\title{
Efficiency of a Two-Item Pre-Screen to Reduce the Burden of Depression Screening in Pregnancy and Postpartum: An IMPLICIT Network Study
}

\author{
Ian M. Bennett, MD, PhD, Andrew Coco, MD, James C. Coyne, PhD, \\ Alex J. Mitchell, MBBS, James Nicholson, MD, MSCE, Ellen Johnson, MD, \\ Michael Horst, PhD, and Stephen Ratcliffe, MD, MSPH
}

Objective: Systems for efficient case finding of women with major depression during pregnancy and postpartum are needed. Here we assess the diagnostic accuracy of a modified 2-item patient health questionnaire (PHQ-2) as a pre-screen in assessing depression.

Methods: Cross-sectional assessments at 15 weeks' gestation $(n=414), 30$ weeks' gestation $(n=$ $334)$, and 6 to 16 weeks postpartum $(n=193)$ among women from a diverse set of races/ethnicities, participating in the IMPLICIT maternal care quality improvement network. The Edinburgh Postnatal Depression Scale score $(\geq 13)$ was used as the criterion measure for the PHQ-2.

Results: A positive 2 -item screen had sensitivity of $93 \%, 82 \%$, and $80 \%$ and specificity of $75 \%, 80 \%$, and 86\% for Edinburgh Postnatal Depression Scale score of $\geq 13$ for assessment at 15 and 30 weeks gestational age and postpartum, respectively. The positive/negative predictive values for the PHQ-2 were 44/98, 24/91, and 30/98 for each time point, respectively. Areas under the receiver operating characteristic curve analysis suggested that 2 -item assessments at each time point had approximately equal diagnostic validity.

Conclusions: Two questions were efficient to rule out depression and reduced the need for further screening of approximately $60 \%$ to $80 \%$ of women, depending on the point in pregnancy or postpartum. A diagnostic interview follow-up of women screening positive is still required. ( $\mathrm{J}$ Am Board Fam Med 2008;21:317-325.)

The identification of perinatal major depression occurring during pregnancy and the first year postpartum is considered a critical goal of the maternal care system but is poorly conducted. ${ }^{1-4}$ Only a minority of women suffering from perinatal depression are identified by health care providers of either maternal or infant care. ${ }^{5-8}$ Although limitations of mental health service delivery play a role in this discrepancy, a major impediment to low rates of depression case finding is the difficulty in the

This article was externally peer reviewed.

Submitted 23 February 2008; revised 28 April 2008; accepted 29 April 2008.

From the Department of Family Medicine and Community Health (IMB, JCC, JN), the Department of Psychiatry (JCC), and the Leonard Davis Institute of Health Economics (IMB), University of Pennsylvania School of Medicine, Philadelphia; the Lancaster General Hospital Department of Family and Community Medicine and Lousie von Hess Medical Research Institute (AC, MH, SR), Lancaster, PA; the Department of Liaison Psychiatry, Leicester General Hospital (AJM), Leicestershire, UK; and the Good Samaritan Family Practice Residency Program (EJ), Lebanon, PA. administration of depression screening in busy clinical settings. ${ }^{9}$ A range of instruments have been used during pregnancy and postpartum, but they generally take at least 5 minutes to complete or rely on adequate literacy of patients for self-administration. In the general primary care setting a 2 -item pre-screen has been validated and has reasonable psychometric properties for ruling out patients at low risk for depression, allowing them to avoid further assessment. ${ }^{10-12}$ This approach has obvious

Funding: This work was supported by a grant from the March of Dimes. IMB was supported by grants from the NICHD (1K23HD048915-01A2) and the NIMH (1R03MH074750-01).

Prior presentation: This work was presented in part at the 2007 North American Primary Care Research Group annual meeting, Vancouver, Canada, October 20 through October 23, 2007.

Conflict of interest: none declared.

Corresponding author: Ian M. Bennett, MD, PhD, Family Medicine and Community Health, University of Pennsylvania School of Medicine, 2nd Floor Gates Pavilion, 3400 Spruce Street, Philadelphia, PA 19104 (E-mail: ian.bennett@ uphs.upenn.edu). 
advantages during pregnancy and has been recommended, with the addition of a third item concerning a request for help, for use by maternal care providers in the British health system. ${ }^{13,14}$ However, to our knowledge this instrument has not been validated in the prenatal and postpartum settings where the normal symptoms of pregnancy can overlap with those of depression.

In this study, we assess the ability of the 2-item version of the patient health questionnaire (PHQ2), with a dichotomous (yes/no) response, to increase the efficiency of screening for risk of major depression during pregnancy and postpartum. Women were evaluated at 2 times during pregnancy and once in the postpartum period using the Edinburgh Postnatal Depression Scale (EPDS) as the criterion. The EPDS is a widely used first step in identifying perinatal depression. ${ }^{15,16}$ The EPDS is most appropriately followed up with an in depth diagnostic interview of women screening positive, but it is efficient in ruling out further assessment for women screening negative; with that caveat in mind, Milgrom et $\mathrm{al}^{17}$ found that the EPDS had a positive predictive value (PPV) of $76 \%$ when measured against Diagnostic and Statistical Manual of Mental Disorders-IV criteria. The goal of the current study was to determine whether the 2-item screening measure could adequately identify women with elevated perinatal depressive symptoms so that a more extensive assessment of women unlikely to have major depression could be avoided.

\section{Methods \\ Setting}

The study sample included patients receiving prenatal and postpartum care from 11 family medicine residency clinical practices in the IMPLICIT (Interventions to Minimize Preterm and Low birth weight Infants through Continuous Improvement Techniques) continuous quality improvement network, which includes rural $(\mathrm{n}=7)$ and urban $(\mathrm{n}=$ 4) sites in Pennsylvania $(n=9)$ and New York $(n=$ 2); institutional review board approval was received from all participating institutions. Women were recruited sequentially to the study from November 2004 through February 2007. Eligibility criteria included English- or Spanish-speaking ability and a singleton intrauterine pregnancy. Women were enrolled in the study at any of 3 time points: twice during pregnancy (15 and 30 weeks' gestation) and once postpartum (6 to 16 weeks after delivery). All eligible women who had complete depression screening data (scores for PHQ-2 and EPDS, see below) for a particular time point were assessed. Women were excluded who had missing depression screening data because of the requirements of the statistical analyses described below. At the 15 week prenatal assessment, 414 of the 785 eligible women had complete data and were used for analysis (52\%), at 30 weeks' gestation 334 of 785 had complete data $(58 \%)$, and at the postpartum assessment 193 of the 274 eligible women had complete data (70\%). Women with missing depression screening data did not differ significantly from those included in the analysis by any demographic variables $(P>$ $.05)$. Women were assessed by physicians or nurses during routine maternal care. Data were then recorded using standardized forms and then entered, after being de-identified, into an Internet-based centralized database for the IMPLICIT network.

\section{Measures of Depression}

Depressive symptomatology was measured using the English and Spanish versions of the EPDS, a 10 -item instrument developed for use in the postpartum period and validated for use during pregnancy, with scores ranging from 0 to $30 .^{18,19}$ The EPDS has been used in a wide range of populations, including low-income women similar to those in the current study. ${ }^{2,20,21}$ A number of cut points have been used and proposed for the identification of perinatal depression, although there are considerable methodological inconsistencies and inadequacies in available studies, precluding a definitive choice among them. ${ }^{22} \mathrm{We}$ have chosen to evaluate EPDS score $\geq 13$ because it has the strongest support for identifying major depression. ${ }^{13} \mathrm{Al}$ though studies are limited, the pooled sensitivity for the identification of major depression in highquality studies at this cut point both in pregnancy and postpartum has been found to be $>95 \%$ with a specificity of $>80 \% .{ }^{13,23}$ The potential pre-screening tool was the 2-item version of the PHQ-2, modified so that response choices were dichotomous (yes/no) rather than 4 ordinally related items. ${ }^{10,24,25}$ Responding yes to either of the items was considered a positive result: "During the past month have you often been bothered by feeling down, depressed, or hopeless?" and "During the past month have you often been bothered by little interest or pleasure in doing things?" Both the 
PHQ-2 and EPDS were initially provided to the patients in printed form as self-response questionnaires. Physicians then orally reviewed the responses to these assessments with patients individually. History of depression was determined by abstracting documentation of previous depression from prenatal and postpartum clinical charts.

\section{Descriptive Variables}

Descriptive variables were selected to aid in the characterization of the sample because of their established associations with depression during pregnancy and postpartum. Demographic variables selected were age, race/ethnicity, educational level, marital status, parity (previous live births), and health insurance status. ${ }^{26-33}$ To differentiate between women who are still in school and those who did not complete school, we divided the $<$ high school completion category based on age: $\leq 18$ years old (potentially still in school) and $>18$ years old (no longer eligible for high school). Self-reported lifetime history of depression is associated with depression during pregnancy and postpartum and was included for evaluation as a potential additional screening item for depression. ${ }^{34-36}$

\section{Statistical Analysis}

Analyses for each of the study time points were conducted independently of the others and so are distinct cross sectional assessments. Bivariate associations between descriptive variables at each time period and a positive EPDS $(\geq 13)$ were assessed using Student's $t$ test and the $\chi^{2}$ statistic, with appropriate extension when assessing variables with more than 2 (dichotomous) categories.

\section{Receiver Operating Characteristic Curve Analysis}

Receiver operating characteristic (ROC) curve analysis was used to evaluate the discrimination capacity of the predictive test (the PHQ-2 alone or with history of depression), or its ability to accurately identify those women in the dichotomized categories (EPDS positive or negative at the cut point of $\geq 13$ ). ${ }^{37}$ ROC curves plot sensitivity (true positive ratio) by 1 -specificity (true negative ratio) for a series of thresholds or cut points established by responses to the instrument items. These thresholds provide information regarding the test characteristics, which can be used to determine the relative usefulness of the test and the specific threshold that maximizes the desired characteristics for a specific clinical setting (emphasizing either the sensitivity or specificity of the instrument). For a 2-item screening instrument, requiring a positive response from only one of the items (cut point $=1$ of 2) maximizes the likelihood of identifying cases (increased sensitivity) but also increases the likelihood of false positives (reduced specificity). Requiring both screening items to be positive to identify a case (cut point $=2$ ) increases the likelihood of missing true positives but also reduces the likelihood of false positives. The area under the ROC curve represents an overall measurement of performance of the screening test, with 1.0 a perfect test and 0.5 representing a test with no discriminating capacity. ${ }^{38}$ All analyses were conducted using SPSS version 15.0 (SPSS, Inc., Chicago, IL).

\section{Results}

\section{Sample Characteristics}

The description of the study sample is included in Table 1. Overall the sample was comprised primarily of young, low-income women with demographic characteristics consistent with that setting. More than half of the participants were between the ages of 19 and 24 years and were white, with approximately one quarter having less than high school education (only one quarter had any college or more); nearly half of the women in the study (45\%) were having their first child, and nearly all with Medicaid insurance or self paid. The distribution of these demographic variables did not vary significantly between the 3 study periods except for race/ethnicity $(P>.05)$; there was a smaller proportion of African-American women in the postpartum sample than in the 2 pregnancy groups.

The percentage of women in the 15- and 30week prenatal and postpartum study periods who were positive by EPDS score for current depressive symptomatology was $17 \%$ (72 of 414 ), $13 \%$ (44 of $334)$, and $5 \%$ (10 of 193), respectively. Of the demographic variables, only Medicaid insurance status was associated with a positive screen with the EPDS. Approximately $30 \%$ of women at each of the 3 time points reported a history of depression. Depression history was significantly associated with a positive EPDS screen for the 15-week and 30week assessments but not the postpartum assessment. The PHQ-2, both alone and when combined with history of depression, was highly associated with a positive EPDS at all 3 of the study assessment points. 
Table 1. Characteristics of the Study Sample of Pregnant and Postpartum Women with Prevalence of Depressive Symptomatology*

\begin{tabular}{|c|c|c|c|c|c|c|}
\hline \multirow[b]{2}{*}{ Descriptive Variables } & \multicolumn{2}{|c|}{15 Week Pregnancy } & \multicolumn{2}{|c|}{30 Week Pregnancy } & \multicolumn{2}{|c|}{ Postpartum } \\
\hline & $\begin{array}{c}\text { Total } \\
(\mathrm{n}=414)\end{array}$ & $\begin{array}{c}\mathrm{EPDS} \geq 13 \\
(\mathrm{n}=72)\end{array}$ & $\begin{array}{c}\text { Total } \\
(\mathrm{n}=334)\end{array}$ & $\begin{array}{c}\mathrm{EPDS} \geq 13 \\
(\mathrm{n}=44)\end{array}$ & $\begin{array}{c}\text { Total } \\
(\mathrm{n}=193)\end{array}$ & $\begin{array}{c}\text { EPDS } \geq 13 \\
(\mathrm{n}=10)\end{array}$ \\
\hline \multicolumn{7}{|l|}{ Demographic variables } \\
\hline \multicolumn{7}{|l|}{ Age } \\
\hline $14-18$ & $53(13)$ & $11(15)$ & $45(14)$ & $8(18)$ & $21(11)$ & 0 \\
\hline $19-24$ & $220(53)$ & $39(54)$ & $172(51)$ & $23(52)$ & $103(53)$ & $5(50)$ \\
\hline $25-29$ & $95(23)$ & $18(25)$ & $76(22)$ & $10(23)$ & $43(22)$ & $3(30)$ \\
\hline $30+$ & $46(11)$ & $4(6)$ & $41(12)$ & $3(7)$ & $26(14)$ & $2(20)$ \\
\hline \multicolumn{7}{|l|}{ Race } \\
\hline Hispanic & $20(5)$ & $4(6)$ & $15(5)$ & $1(2)$ & $10(5)$ & 0 \\
\hline African-American & $125(30)$ & $27(38)$ & $88(26)$ & $15(34)$ & $41(21)$ & $2(20)$ \\
\hline White & $258(62)$ & $39(54)$ & $226(68)$ & $28(64)$ & $137(71)$ & $8(80)$ \\
\hline Other & $11(3)$ & $2(3)$ & $5(2)$ & 0 & $5(3)$ & 0 \\
\hline \multicolumn{7}{|l|}{ Education } \\
\hline$<$ High school & $94(23)$ & $20(28)$ & $76(23)$ & $16(36)$ & $43(22)$ & $1(10)$ \\
\hline Still in high school & $42(10)$ & $6(8)$ & $36(11)$ & $6(14)$ & $17(9)$ & 0 \\
\hline High school or equivalent & $172(42)$ & $29(40)$ & $136(41)$ & $14(32)$ & $89(46)$ & $7(70)$ \\
\hline Any college or more & $106(26)$ & $17(24)$ & $86(26)$ & $8(18)$ & $44(22)$ & $2(20)$ \\
\hline \multicolumn{7}{|l|}{ Parity (previous live births) } \\
\hline 0 & $185(45)$ & $27(38)$ & $142(43)$ & $14(32)$ & $81(42)$ & $3(30)$ \\
\hline 1 & $117(28)$ & $23(32)$ & $89(27)$ & $16(36)$ & $54(28)$ & $4(40)$ \\
\hline $2-5$ & $112(27)$ & $22(31)$ & $103(31)$ & $14(32)$ & $58(30)$ & $3(30)$ \\
\hline \multicolumn{7}{|l|}{ Insurance } \\
\hline \multicolumn{7}{|l|}{ Medical assistance } \\
\hline Self-pay & $375(91)$ & $70(97)$ & $301(90)$ & $42(96)$ & $168(87)$ & $10(100)$ \\
\hline Private & $39(9)$ & $2(3)$ & $33(10)$ & $2(5)$ & $25(13)$ & 0 \\
\hline \multicolumn{7}{|l|}{ Depression measures } \\
\hline History of depression & $122(30)$ & $45(63)$ & $97(29)$ & $23(52)$ & $54(28)$ & $5(50)$ \\
\hline Two-item screen positive & $151(37)$ & $67(93)$ & $94(28)$ & $36(82)$ & $33(17)$ & $8(80)$ \\
\hline Two-item screen and history of depression & $42(50)$ & $70(97)$ & $148(44)$ & $41(93)$ & $54(37)$ & $9(90)$ \\
\hline
\end{tabular}

*Edinburgh Postnatal Depression Scale score $\geq 13$.

All values provided a $\mathrm{n}(\%)$. Bolded values indicate significant association $\left(P<.05\right.$; Pearson's $\chi^{2}$ or Fisher's exact test if cells contain $\leq 5)$.

\section{Diagnostic Accuracy}

The characteristics of the PHQ-2 for identifying positive EPDS cases, alone and in combination with a history of depression, are shown in Table 2 and Figure 1. The cut points for the combined PHQ-2 and history of depression screening instrument are labeled as $1 / 2$ (positive for either the PHQ-2 or history of depression) and 2 (positive for both PHQ-2 and history of depression). The overall performance of the measure as determined by the area under the ROC curve were similar across the 3 study points and had similar point estimates and overlapping confidence intervals when the
PHQ-2 was used alone or in combination with the history of depression (Table 2, column 2).

\section{(1) Screening at week 15 (estimated gestational age [EGA])}

The PHQ-2 allowed the detection of 67 of 72 women with depression, defined by a score of 13 or above on the EPDS, with a sensitivity of $93.1 \%$. Specificity was $75.1 \%$, with a PPV of $44 \%$ and negative predictive value (NPV) $98 \%$. When the PHQ-2 was combined with a history of depression, sensitivity was improved to $97.2 \%$ but at the expense of specificity $(61.1 \%)$. 
Table 2. Diagnostic Validity of the Two-Item Patient Health Questionnaire for the Edinburgh Postnatal Depression Scale*

\begin{tabular}{|c|c|c|c|c|c|}
\hline Variable(s) & AUROC Curve (95\% CI) & Sensitivity (\%) & Specificity (\%) & PPV (\%) & NPV (\%) \\
\hline \multicolumn{6}{|l|}{$\mathrm{EPDS} \geq 13$} \\
\hline \multicolumn{6}{|l|}{15 weeks' EGA } \\
\hline PHQ-2 & $0.80(0.69-0.91)$ & 93 & 75 & 44 & 98 \\
\hline PHQ-2 and history of depression & $0.85(0.76-0.94)$ & & & & \\
\hline Cut point $1 / 2$ & & 97 & 61 & 59 & 91 \\
\hline Cut point 2 & & 58 & 92 & 21 & 99 \\
\hline \multicolumn{6}{|l|}{30 weeks' EGA } \\
\hline PHQ-2 & $0.81(0.74-0.88)$ & 82 & 80 & 24 & 91 \\
\hline PHQ-2 and history of depression & $0.85(0.76-0.94)$ & & & & \\
\hline Cut Point $1 / 2$ & & 93 & 63 & 42 & 91 \\
\hline Cut Point 2 & & 41 & 91 & 22 & 98 \\
\hline \multicolumn{6}{|l|}{ Postpartum } \\
\hline PHQ-2 & $0.83(0.68-0.98)$ & 80 & 86 & 30 & 98 \\
\hline PHQ-2 and history of depression & $0.83(0.70-0.97)$ & & & & \\
\hline Cut point $1 / 2$ & & 90 & 67 & 44 & 96 \\
\hline Cut point 2 & & 60 & 96 & 11 & 99 \\
\hline
\end{tabular}

*Edinburgh Postnatal Depression Scale (EPDS) score $\geq 13$.

PHQ-2, 2-item Patient Health Questionaire; AUROC, area under the receiving operator characteristic; PPV, positive predictive value; NPV, negative predictive value; EGA, estimated gestational age; Cut point 1/2, one of 2 screening items positive; Cut point 2, both screening items positive.

\section{(2) Screening at week 30 (EGA).}

The PHQ-2 facilitated the detection of 36 of 44 women with depression, defined by a score of 13 or above on the EPDS, with a sensitivity of $81.8 \%$. Specificity was $80.0 \%$, with a PPV of $38 \%$ and NPV 97\%. When the PHQ was combined with a history of depression, sensitivity was improved to 93.2\% but at the expense of specificity $(63.1 \%)$.

\section{(3) Postpartum Screening (6 to 16 weeks after birth)} The PHQ-2 identified 8 of 10 women with postpartum depression, defined by a score of 13 or

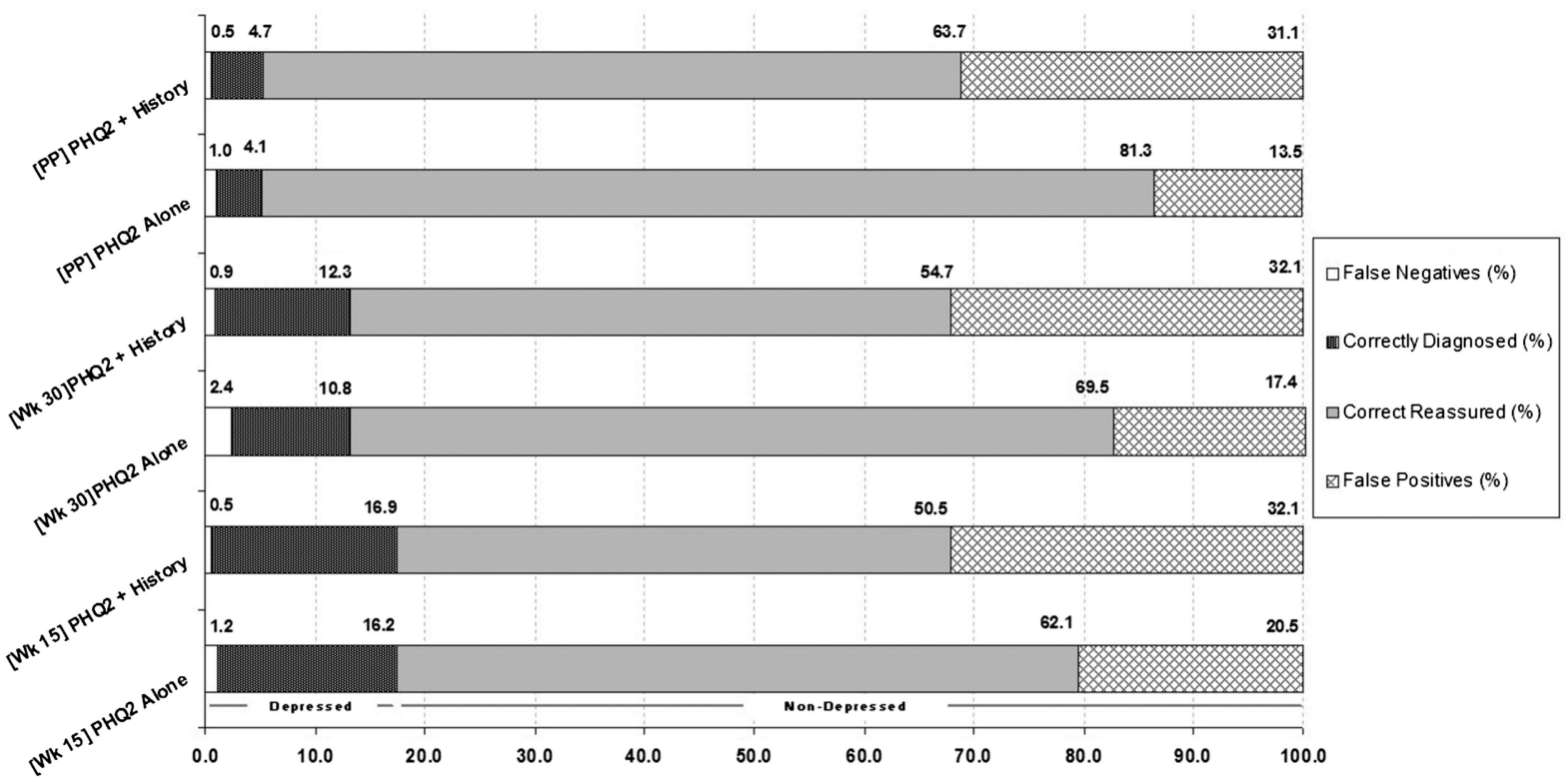

Figure 1. Proportionate accuracy of the PHQ-2 versus EPDS (12 vs 13) per 100 unselected patients. 
above on the EPDS, with a sensitivity of $80.0 \%$. Specificity was $85.8 \%$, with a PPV of $24 \%$ and NPV 99\%. When the PHQ was combined with a history of depression sensitivity was improved to $90.0 \%$ but at the expense of specificity (67.2\%).

\section{Discussion}

In this analysis of women during pregnancy and postpartum we determined that pre-screening with the 2-item PHQ generally had good sensitivity and specificity for identifying women with positive scores $\geq 13$ on the Edinburgh Postnatal Depression Scale. The addition of a question regarding history of depression improved the sensitivity, at the expense of specificity, and the PPV of this screening. Out of 100 unselected cases seen at 15 weeks, the PHQ-2 alone would help correctly detect 16 out of 17 depressed patients (missing only one patient) and correctly reassure 62 out of 83 patients, falsely diagnosing 21. Similarly, out of 100 unselected patients seen postpartum, the PHQ-2 alone would help correctly identify 4 out of 5 depressed patients (the prevalence is $5 \%$ in this case) and correctly reassure 81 out of 95 patients, misidentifying 13 . This demonstrates that when the PHQ-2 is negative it has a $97 \%$ to $99 \%$ chance of accurately ruling out depression with very few false negatives. Thus, this rapid screening procedure may be useful as part of a multistage case finding strategy to rule out women who would otherwise require further timeconsuming assessment for the presence of maternal depression.

Based on the rate of positive 2-item screens in the current study, $60 \%$ to $80 \%$ of the women being assessed (depending on the particular point in pregnancy and postpartum) can be ruled out with high certainty of not having depression. When the history of depression item is added, the number of women quickly ruled out is slightly higher but at a cost of approximately $10 \%$ in case-finding accuracy. It is important to keep in mind that even when the PHQ-2 is negative and the NPV is $90 \%$ or higher, it is possible that these women actually have major depression. For this reason we suggest that a negative screen should not overrule clinical concern and that anyone judged to be symptomatic clinically but with a negative pre-screen or screen for depression should have a further concurrent or follow-up examination. It may be somewhat surprising that adding history of depression questions did not substantially improve the performance of prescreening, particularly given the recognition that depression is a recurrent episodic disorder. ${ }^{39}$ One explanation is that the women in our sample were young enough that they were still experiencing their first episode of depression. A second reason might be that the PHQ-2 alone has reached a ceiling regarding rule-out accuracy. A third possible reason questions the validity of simple selfreport questions for assessing history without a basis for explaining the questions or probing the responses that an interview provides, a finding that has been reported elsewhere. ${ }^{40}$

Importantly, we have shown that the modified PHQ-2 used here functions well as a first prescreening step in early and late pregnancy and postpartum. By efficiently ruling out those who do not have depression, resources can be targeted at the women at highest risk of a major depressive disorder. However, the value of this strategy depends on diagnostic follow-up of the women registering positive with both the pre-screening and the EPDS. A positive score on the EPDS is by itself an inadequate basis for clinical decision making and, if used as such, scarce resources might be used on the treatment of women who are not truly depressed and in need of help. There is some evidence that busy primary care clinicians are inclined not to follow up on the results of screening questionnaires, instead accepting a positive score on a screening questionnaire as a sufficient basis for diagnosis. ${ }^{41}$ It would be unfortunate if the introduction of a pre-screen further encouraged this practice; before recommending this pre-screening strategy clinically it would be important to determine that it would not be used inappropriately.

Although we assessed the PHQ-2 as a prescreen it has been recommended for use alone as a screening instrument in general primary care practice. ${ }^{10}$ It is possible that this instrument, without the use of the EPDS, has sufficient accuracy to identify women at risk (or those not at risk) for depression during pregnancy and postpartum. Currently, the EPDS is well validated and highly accepted in perinatal settings, but little work has justified the use of the PHQ-2 to date. A 3-question approach (essentially the PHQ-2 plus a help question) has been recommended by the British health system's National Institute for Clinical Excellence guidelines but has never been formally evaluated in perinatal settings. ${ }^{12}$ In one study, Olson et $\mathrm{al}^{42}$ 
found that $17 \%$ of 1398 mothers answered positively to at least one question of the PHQ-2, although validity was not reported. Recently, Dubowitz et $\mathrm{al}^{43}$ adapted the PHQ-2 into the "Parent Screening Questionnaire." The authors evaluated this questionnaire among 216 mothers in a primary care clinic compared with the Beck Depression Inventory II completed 2 months later. When a positive response to either or both of the 2 questions was considered, the sensitivity was $74 \%$, the specificity was $80 \%$, and the NPV was $95 \%$, but the PPV was only $36 \%$.

An unresolved issue is how many of those who screen positive actually are willing to accept professional help. In one study, ${ }^{44}$ only $30 \%$ of those who screened positive agreed to be contacted for further help. In a second study, ${ }^{45}$ only $23 \%$ of high scorers on the Beck Depression Inventory took up the offer for psychological therapy and 10\% agreed to medication. This highlights that screening alone is rarely sufficient for improvements in quality of care, and enhanced detection should be paired with enhanced treatment and follow-up. ${ }^{46}$

A number of limitations of the current study should be reviewed. First, we use as a criterion measure the EPDS screening instrument rather than the clinical diagnosis of depression. However, the goal of the current analysis was to reduce the burden of screening for depression through a prescreen step, not to diagnose major depressive disorder. Because the formal diagnosis of depression requires significant diagnostic expertise and the investment of valuable clinical time, the rapid identification of women at risk for depression (and not at risk) is critical to the efficient delivery of maternal care. Another limitation of the current study is that the women included were primarily low income as indicated by the high rate of Medicaid insurance. The findings from this study may not be generalizable to higher-income and other less vulnerable populations. However, women with low incomes have higher risk for depressive symptomatology and are less likely to receive mental health services and so are a particularly important group to target for screening. ${ }^{4,47}$ One recent study ${ }^{48}$ found that the sensitivity of the PHQ-2 for a positive EPDS score was lower for women with a high school education or less than for those who went on beyond high school. We did not find any difference in sensitivity across levels of educational attainment in our sample. This difference may be because of our use of a simpler dichotomous yes/no response choice for the PHQ-2 and a follow-up oral review of the items, which reduces concerns of low education- and literacy-related inaccuracy of a written and more complex instrument. ${ }^{11}$ Finally, although we had complete data for more than $50 \%$ of the women eligible for analysis at each of the time points, it is possible that missing data were related to depression risk status. For example, women with more obvious symptoms may have been either more or less likely to have both the pre-screen (PHQ-2) and screening (EPDS) conducted (both were required to be included in the analysis). Because of the protocol-driven system of data collection in this study, we believe that it is unlikely that any such bias was widespread enough to greatly influence our findings.

Based on the results of these analyses it is reasonable for maternal care providers who are currently using or are planning to use the EPDS to use the PHQ-2 pre-screen, with simplified response items (yes/no), as an initial component of a multistep case-finding strategy for depression during pregnancy and postpartum. Although this approach should not overrule clinical concern, women who answer "no" to both of the questions in the PHQ-2 can, with high confidence, avoid the longer EPDS and a diagnostic clinical interview. Women who answer "yes" to either of these items should proceed to the EPDS, followed by an in-depth clinical interview for women who have a positive EPDS $(\geq 13)$. Although further research is needed, we recommend that the screening process make use of a direct oral interview rather than relying entirely on a written questionnaire to elicit or review screening questions to avoid error caused by low education and literacy.

Thanks to all the contributing members of the IMPLICIT network and the Family Medicine Educational Consortium for contributions that made this work possible.

\section{References}

1. Buist A, Bilszta J, Barnett B, et al. Recognition and management of perinatal depression in general practice-a survey of GPs and postnatal women. Aust Fam Physician 2005;34:787-90.

2. Chaudron LH, Szilagyi PG, Kitzman HJ, Wadkins HI, Conwell Y. Detection of postpartum depressive symptoms by screening at well-child visits. Pediatrics Mar 2004;113(3 Pt 1):551-8.

3. Cohen LS, Nonacs R, Viguera AC, Reminick A. 
Diagnosis and treatment of depression during pregnancy. CNS Spectr 2004;9:209-16.

4. Miranda J, Azocar F, Komaromy M, Golding JM. Unmet mental health needs of women in publicsector gynecologic clinics. Am J Obstet Gynecol 1998;178:212-7.

5. Healthy People 2010: Understanding and improving health. Washington DC: Department of Health and Human Services; 2000.

6. Bagedahl-Strindlund M, Borjesson KM. Postnatal depression: a hidden illness. Acta Psychiatr Scand 1998;98:272-5.

7. Coates AO, Schaefer CA, Alexander JL. Detection of postpartum depression and anxiety in a large health plan. J Behav Health Serv Res 2004;31:117-33.

8. Thio IM, Browne MAO, Coverdale JH, Argyle N. Postnatal depressive symptoms go largely untreated: a probability study in urban New Zealand. Soc Psychiatry Psychiatr Epidemiol 2006;41:814-8.

9. Seehusen DA, Baldwin LM, Runkle GP, Clark G. Are family physicians appropriately screening for postpartum depression? J Am Board Fam Med 2005; 18:104-12.

10. Berg AO, Allan JD, Frame PS, et al. Screening for depression: USPSTF recommendations and rationale. Ann Intern Med 2002;136:760-4.

11. Arroll B, Khin N, Kerse N. Screening for depression in primary care with two verbally asked questions: cross sectional study. BMJ 2003;327:1144-6.

12. Mitchell AJ, Coyne JC. Do ultra-short screening instruments accurately detect depression in primary care? A pooled analysis and meta-analysis of 22 studies. Br J Gen Pract 2007;57:144-51.

13. Gaynes BN, Gavin N, Meltzer-Brody S, et al. Perinatal depression: prevalence, screening accuracy, and screening outcomes. Rockville (MD): Agency for Healthcare Research and Quality; 2005:119.

14. Antenatal and postnatal mental health clinical management and service guidance (NICE clinical guideline \#45). United Kingdom: National Health Service; 2007.

15. Boyd RC, Le HN, Somberg R. Review of screening instruments for postpartum depression. Arch Womens Ment Health 2005;8:141-53.

16. Eberhard-Gran M, Eskild A, Tambs K, Opjordsmoen S, Samuelsen SO. Review of validation studies of the Edinburgh postnatal depression scale. Acta Psychiatr Scand 2001;104:243-9.

17. Milgrom J, Ericksen J, Negri L, Gemmill AW, Shakespeare J. Evaluation of screening for postnatal depression against the NSC handbook criteria. Aust N Z J Psychiatry 2005;39:833-9.

18. Cox JL, Chapman G, Murray D, Jones P. Validation of the Edinburgh Postnatal Depression Scale (EPDS) in non-postnatal women. J Affect Disord 1996;39:185-9.

19. Cox JL, Holden JM, Sagovsky R. Detection of postnatal depression. Development of the 10-item Edin- burgh Postnatal Depression Scale. Br J Psychiatry 1987;150:782-6.

20. Areias ME, Kumar R, Barros H, Figueiredo E. Correlates of postnatal depression in mothers and fathers. Br J Psychiatry 1996;169:36-41.

21. Bennett I, Switzer J, Aguirre A, Evans K, Barg F. "Breaking it down": patient-clinician communication and prenatal care among African-American women of low and higher literacy. Ann Fam Med 2006;4:334-40.

22. Matthey S, Henshaw C, Elliott S, Barnett B. Variability in use of cut-off scores and formats on the Edinburgh Postnatal Depression Scale - implications for clinical and research practice. Arch Womens Ment Health 2006;9:309-15.

23. Murray D, Cox JL. Screening for depression during pregnancy with the Edinburgh Depression Scale (EPDS). J Reprod Infant Psychol 1990;8:99-107.

24. Kroenke K, Spitzer RL, Williams JBW. The Patient Health Questionnaire-2: validity of a two-item depression screener. Med Care 2003;41:1284-92.

25. Whooley MA, Avins AL, Miranda J, Browner WS. Case-finding instruments for depression. Two questions are as good as many. J Gen Int Med 1997;12: 439-45.

26. Altshuler LL, Hendrick V, Cohen LS. Course of mood and anxiety disorders during pregnancy and the postpartum period. J Clin Psychiatry 1998; 59(Suppl 2):29-33.

27. Berenson AB, Breitkopf CR, Wu ZH. Reproductive correlates of depressive symptoms among lowincome minority women. Obstet Gynecol 2003;102: $1310-7$.

28. Gotlib IH, Whiffen VE, Mount JH, Milne K, Cordy NI. Prevalence rates and demographic characteristics associated with depression in pregnancy and the postpartum. J Consult Clin Psychol 1989;57:26974.

29. Hobfoll SE, Ritter C, Lavin J, Hulsizer MR, Cameron RP. Depression prevalence and incidence among inner-city pregnant and postpartum women. J Consult Clin Psychol 1995;63:445-3.

30. Howell E, Mora P, Leventhal H. Correlates of early postpartum depressive symptoms. Matern Child Health J 2006;10:149-57.

31. Howell EA, Mora PA, Horowitz CR, Leventhal H. Racial and ethnic differences in factors associated with early postpartum depressive symptoms. Obstet Gynecol 2005;105:1442-50.

32. Kuo WH, Wilson TE, Holman S, Fuentes-Afflick E, O'Sullivan MJ, Minkoff H. Depressive symptoms in the immediate postpartum period among Hispanic women in three US cities. J Immigr Health 2004;6: 145-53.

33. Larsson D, Lager I, Nilsson PM. Socio-economic characteristics and quality of life in diabetes mellitus-relation to metabolic control. Scand J Public Health 1999;27:101-5. 
34. Bernazzani O, Saucier JF, David H, Borgeat F. Psychosocial predictors of depressive symptomatology level in postpartum women. J Affect Disord 1997;46: 39-49.

35. Bernazzani O, Saucier JF, David H, Borgeat F. Psychosocial factors related to emotional disturbances during pregnancy. J Psychos Res 1997;42:391-402.

36. O'Hara MW, Schlechte JA, Lewis DA, Wright EJ. Prospective study of postpartum blues. Biologic and psychosocial factors. Arch Gen Psychiatry 1991;48: 801-6.

37. Justice A, Covinsky K, Berlin J. Assessing the generalizability of prognostic information. Ann Intern Med 1999;130:515-24.

38. Hanley J, McNeil B. The meaning and use of the area under a receiver operating characteristic curve. Radiology 1982;143:29-36.

39. Klinkman MS, Schwenk TL, Coyne JC. Depression in primary care - more like asthma than appendicitis: The Michigan Depression Project. Can J Psychiatry 1997;42:966-73.

40. Coyne JC, Thompson R, Racioppo MW. Validity and efficiency of screening for history of depression by self-report. Psychol Assess 2001;13:163-70.

41. Valenstein M, Dalack G, Blow F, Figueroa S, Standiford C, Douglass A. Screening for psychiatric ill- ness with a combined screening and diagnostic instrument. J Gen Int Med 1997;12:679-85.

42. Olson AL, Dietrich AJ, Prazar G, Hurley J. Brief maternal depression screening at well-child visits. Pediatrics 2006;118:207-16.

43. Dubowitz H, Feigelman S, Lane $W$, et al. Screening for depression in an urban pediatric primary care clinic. Pediatrics 2007;119:435-43.

44. Carter FA, Carter JD, Luty SE, Wilson DA, Frampton CMA, Joyce PR. Screening and treatment for depression during pregnancy: a cautionary note. Aust N Z J Psychiatry 2005;39:255-61.

45. Horowitz JA, Cousins A. Postpartum depression treatment rates for at-risk women. Nurs Res 2006; 55(2 Suppl):S23-S27.

46. Gilbody SD, Sheldon TD, House ADM. Screening and case-finding instruments for depression: a metaanalysis. CMAJ 2008;178:997-1003.

47. Miranda J, Chung JY, Green BL, et al. Treating depression in predominantly low-income young minority women: a randomized controlled trial. JAMA 2003;290:57-65.

48. Cutler CB, Legano LA, Dreyer BP, et al. Screening for maternal depression in a low education population using a two item questionnaire. Arch Womens Ment Health 2007;10:277-83. 